\title{
The Effect of Limbal Autograft in Recurrence of Pterygium
}

\author{
Suleyman Ciftci ${ }^{*}$, Eyup Dogan${ }^{1}$, Leyla Ciftci², Ozlem Demirpence ${ }^{3}$ \\ ${ }^{1}$ Department of Ophthalmology, Diyarbakır Training and Research Hospital, Diyarbakır, Turkey \\ ${ }^{2}$ Department of Cardiology, Faculty of Medicine, Dicle University, Diyarbakır, Turkey \\ ${ }^{3}$ Department of Biochemistry, Tunceli State Hospital, Tunceli, Turkey \\ Email: ${ }^{*}$ ciftci1977@hotmail.com
}

Received 11 March 2015; accepted 31 July 2015; published 4 August 2015

Copyright (C) 2015 by authors and Scientific Research Publishing Inc.

This work is licensed under the Creative Commons Attribution International License (CC BY).

http://creativecommons.org/licenses/by/4.0/

(c) (i) Open Access

\section{Abstract}

Background: Assessing the effect of limbal autograft shifting in recurrence of pterygium. Methods: This single-center study was carried out in a tertiary health facility. A review of data on consecutive patients who underwent pure limbal autografts shifting after pterygium resection was done. In all the cases, the pterygia extended at least $3 \mathrm{~mm}$ beyond the limbus. The resected each limbal grafts included a width of $1.5 \mathrm{~mm}$ and a length of 2 or $3 \mathrm{~mm}$ of limbus and a depth of $250 \mu \mathrm{m}$. Schmer test was performed at the eighth month postoperatively. Pterygium recurrence was accepted endpoint of the study. One patient had recurrent pterygium, whereas the others had primary pterygium. Patients with other ocular surface diseases or ocular pathology and, patients who discontinued follow-up visits were excluded from the study. Results: The study included 10 patients, with 5 males and 5 females. Median age of the patients was 40 (25 - 70). Follow-up was conducted for a minimum of 8 months for patients with recurrence and at least for $\mathbf{1 6}$ months for non-recurrent cases. Recurrence was observed in 6 patients out of 10, in one patient, atypia was reported and excluded from the study. Four recurrent patients experienced decreased levels of tears. The rest one patient with recurrence had not any tear abnormality. The remaining 4 patients responded well to the surgery. Because of the high recurrence rate, it was decided to terminate the study. Conclusions: Limbal autografts shifting alone is not an appropriate treatment for primary pterygium because of the high recurrence rate.

\section{Keywords}

Limbal Autograft, Recurrence, Pterygium

\footnotetext{
${ }^{*}$ Corresponding author.
} 


\section{Article Summary}

Pterygium is a complex disease and the benefits of limbal graft are limited when working alone. As such, pterygium may be defined as a final common formation of some interactive instabilities that constitute fibrovascular tissue. Dysfunction of the limbus affects the results of this study favorably whereas without excised tenon's layer affect the results of this study unfavorably. Currently conjunctival-limbal autografts combining with tenon's layer removal seem to manage many factors that actively participate in the formation of pterygium.

This study has limited number of participants and lack of control group; however it proved several points and complimentary indications such as atypia, asymmetric dry eye, effects of limbus in recurrence and in multiple pterygium and effect of tenon's layer comparable to limbal stem cells. These are vital clues for the causes of recurrence.

\section{Introduction}

Pterygium represents a pathologic condition occurring more frequently in certain populations. Its incidence varies greatly with different geographical zones. A lot of factors such as genetic, environmental, ocular surface instability, limbal deficiency and atypia play an active role either individually or synergistically in the development of pterygium. In general, pterygium may be defined as an end-product of some interactive instabilities that constitute the fibrovascular tissue [1].

Some of the existing approaches to the treatment of pterygium were evolved on the basis of the knowledge acquired from recent etiopathogenetic and clinical studies that emphasized the role of limbus [2]-[5]. Therefore, especially conjunctival-limbal autograft became the more preferred surgery, with a relatively shorter operative time and a low rate of recurrence as well as comparable to adjuvants [6]-[15].

Conceptually, the number of recurrences of pterygium could possibly be reduced by including the limbus in the conjunctival autograft used in the surgical technique because conjunctival-limbal autografts make more physiological reparation [8]. However, there is no clear explanation on the additive successful result of conjunctivallimbal autograft that is ensured by interaction effect of the grafts with each other or is ensured by pure effect of limbal autograft [6]. Pure limbal autograft, as a technique for pterygium surgery appears to be an appropriate model to test the knowledge acquired from recent studies and to determine the utility of this technique in the management of all forms of pterygium (etc., primary or recurrent) clinically. Even, Shimazaki et al. experienced a similar technique in recurrent pterygium previously and reported favorable results [5]. They used limbal autograft transplantation. Superior limbal tissue was taken with conjunctival flap and transferred to the excised area. However, they attributed favorable results to limbal autograft. They used conjunctival flap conjunction with limbus. However, we know that autologous conjunctival transplantation has favorable impact on recurrence [6] [9] [10]. The main difference between our technique and Shimazaki's is that we balance favorable impact of conjunctiva by unexcised tenon's tissue.

In the preliminary study, the effect of pure limbus in recurrence of pterygium was assessed in order to understand the exact mechanism of success based upon limbal autograft coupled with decreased rates of recurrence.

\section{Materials and Methods}

This single-center study was carried out in a tertiary health facility. A review of data on consecutive patients who underwent pure limbal autografts shifting after pterygium resection during the period from May 2011 to August 2011 was done. The measurement of the rate of recurrence is the key factor of this study and pterygium recurrence was accepted endpoint of the study. A true recurrence was defined as a fibro-vascular ingrowth of 1.5 mm or more across the limbus with conjunctival drag [16].

Informed consent was obtained from all the patients who participated in this study. This study was approved by The Institutional Review Board of Batman State Hospital (Batman, Turkey). The tenets of the Declaration of Helsinki were followed. Patient consent was obtained for use of figures accompanying this paper. One of the patients had recurrent pterygium, whereas the others had primary pterygium. Patients with a clinically apparent ocular surface disease or ocular pathology, and patients who discontinued follow-up visits were excluded from the study. None of the patients had previously undergone any ocular procedures except for pterygium surgery in one of the patients.

In all the cases, the pterygia extended at least $3 \mathrm{~mm}$ beyond the limbus and in stage IV or V. All the opera- 
tions were performed by the same surgeon who experienced in ocular surface surgery including limbal autografting and pterygium surgery. The surgical technique is shown in Figure 1. The surgeries were performed under sub-tenon's anesthesia with $2 \%$ lidocaine. After the injection of lidocaine under the conjunctiva in the body of the pterygium, dissection was performed from the head toward the body (Figure 1, top right and top left). The corneal defect was shaved for any residual tissue using a blade. The limbal grafts including stem cells were harvested from the upper and the lower adjacent healthy corneal limbus (Figure 1, bottom left). The resected each limbal grafts included a width of $1.5 \mathrm{~mm}$ and a length of 2 or $3 \mathrm{~mm}$ of limbus and a depth of $250 \mu \mathrm{m}$. The harvested limbal grafts' length was adjusted according to the excised pterygium size in the head portion which was between 4 or $6 \mathrm{~mm}$. The adjacent episcleral and conjunctival tissue and tenon's layer in harvested limbal graft was maintained and both the adjacent conjunctiva and tenon's layer was released from episclera in superior and inferior portions behind the limbus. Once these grafts were shifted to the excised region of pterygium while maintaining limbus to limbus orientation, the adjacent tissue dislocated to the midline with the limbal graft. Then the grafts and adjacent episcleral and conjunctival tissue were secured with interrupted 10.0 nylon sutures. The conjunctiva was closed with 10.0 nylon sutures, leaving no bare sclera (Figure 1, bottom right). The donor site was left to epithelialize without closure of the defect. The duration of the procedure was approximately one hour. A bandage closure was applied postoperatively to all patients for a week. The specimens were sent for pathologic analysis. Sutures were removed after one month.

Postsurgically, oral analgesia was administered for the first day, topical dexamethasone solutions for at least 45 days on a tapered regimen until conjunctival injection resolved, moxifloxacin for two weeks and lubrication for one month. Schirmer test was performed at the eighth month postsurgery. Follow up was made in postoperative first day, first week, first month, and later every month. At each visit macrophotography of the eye and slit-lamp examination were undertaken. Patients who failed to return for the recommended follow-up appointments were telephoned to encourage compliance with follow-up.

\section{Results}

This study was conducted on a total of 10 patients, consisting of 5 males and 5 females. Median age of the patients was 40 (25 - 70). The details of the patients are presented in Table 1. Primary pterygium was found in eight patients, recurrent pterygium in one patient and multiple pterygium in one patient. All the patients were followed-up for a minimum period of eight months. In one of the patients (Table 1, Patient No. 6), atypia was reported in the pathology report, all other specimens were consistent with pterygium. In the patient whom atypia was reported recurrence was observed very early. According to methodology this patient was excluded from the study. All the other recurrences began to develop after three months and got completed in less than eight months.
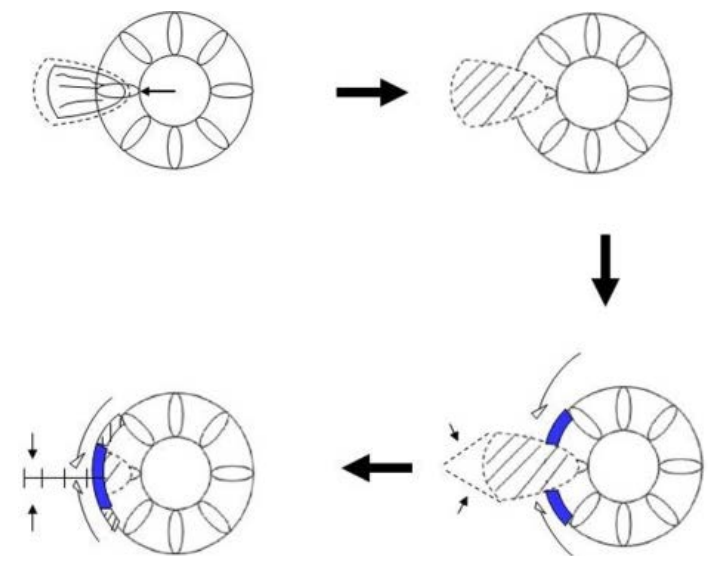

Figure 1. Top right. Dissection was continued from the head toward the body; top left. The corneal defect was shaved for any residual tissue using a blade; bottom left. The limbal grafts including stem cells were harvested from the upper and the lower adjacent healthy corneal limbus; bottom right. The grafts were shifted to the excised region of pterygium maintaining limbus to limbus orientation. The grafts were secured with interrupted 10.0 nylon and conjunctiva was closed with 10.0 nylon sutures, leaving no bare sclera. 
Table 1. Clinical data of the patients.

\begin{tabular}{ccccccccc}
\hline $\begin{array}{c}\text { Patient } \\
\text { No. }\end{array}$ & $\begin{array}{c}\text { Age } \\
\text { (Yr) }\end{array}$ & Sex & $\begin{array}{c}\text { Follow-Up } \\
\text { (month) }\end{array}$ & Presentation & $\begin{array}{c}\text { Recurrence and time } \\
\text { (month) }\end{array}$ & Atypia & Schmer & Side \\
\hline 1 & 65 & M & 16 & Multiple, primary & No & No & $24 / 24$ & R \\
2 & 44 & F & 16 & Primary & No & No & $30 / 15$ & R \\
3 & 28 & M & 8 & Primary & Yes, between 3 to 8 & No & $30 / 30$ & R \\
4 & 60 & F & 16 & Recurrent & No & No & $25 / 25$ & $\mathrm{~L}$ \\
5 & 40 & F & 8 & Primary & Yes, between 3 to 8 & No & $3 / 25$ & $\mathrm{R}$ \\
6 & 26 & F & 8 & Primary & Yes, in 1,5 & Yes & $30 / 30$ & $\mathrm{R}$ \\
7 & 30 & M & 8 & Primary & Yes, between 3 to 8 & No & $35 / 15$ & $\mathrm{~L}$ \\
8 & 32 & M & 8 & Primary & Yes, between 3 to 8 & No & $19 / 30$ & $\mathrm{R}$ \\
9 & 25 & M & 16 & Primary & No & No & $35 / 35$ & $\mathrm{~L}$ \\
10 & 70 & F & 8 & Primary & Yes, between 3 to 8 & No & $35 / 15$ & $\mathrm{~L}$ \\
\hline
\end{tabular}

M; male, F; female, R, right; L, left; m, months.

Hence, the follow-up was limited to eight months among all the five recurrent cases. In total, recurrence was observed in five patients with a recurrence rate of 55.5\%. In four of the recurrent cases, the quantity of tears decreased on the region of recurrence and one of these patients is presented in Table 1 as fifth patient having dry eye. Artificial tear and cyclosporine were prescribed to the patients. After medical treatment these patients were free from eye complaint and declined further operation. Because the rate of recurrence following this surgical approach was high within the eight months of follow-up, the study was terminated and no new patients were enrolled to the study.

The follow-up period was extended to at least 16 months for 4 patients with no recurrence with a surgical success rate of $44.5 \%$. One of these patients, presented in Table 1 as fourth patient, had recurrent pterygium and had received conjunctival autograft. Recurrence developed one month after the surgery (Figure 2). In this patient, the previous recurrence appeared to be caused by dysfunction of the limbus. Another patient presented in Table 1 as first patient had multiple pterygia and responded well to limbal shifting surgery. The cause of multiple pterygia in this patient appears to be due to dysfunction of the limbus.

\section{Discussion}

The effect of pure limbal autograft shifting in recurrence of pterygium was assessed in this retrospective study. It was found that the rate of recurrence of this technique was $55.5 \%$ in a sample size of 9 patients. Hence, it was concluded that the effect of limbus shifting in this study was not better than primary pterygium excision and, the enrollment of patients into the study was discontinued. In addition, no control group was formed for this study.

In accordance with the criteria set out by Singh, recurrences of pterygial growth necessitate an extended time, hence exact time of recurrence could not be determined [16]. Instead the time from the initial stage to the completion of pterygial recurrence was determined. Survival curve analysis indicated that there existed 50\% chance of recurrence of pterygium within the first 120 days, and a 97\% chance within 12 months of its removal [17]. Follow-up period was determined based on this information. Follow-up of the patients with recurrence was discontinued when full formation stage occurred and follow-up extended up to 16 months for patients without recurrence.

In this study, an alternative treatment for pterygium is not being proposed; hence the complications associated with the surgery were not reported. However, a new pterygium surgery technique to test the effect of pure limbal autograft in recurrence of pterygium was used. As determined in this study, treatment modalities in pterygium fail when a single reason is considered. Responding well to limbal shifting surgery in this study, the response of the two the patients: one with recurrent pterygium and the other with multiple pterygia is attributed to dysfunction of the limbus. Dysfunction of the limbus was found to affect the results of this study favorably. Results in dysfunction of the limbus were consistent with the findings of Shimazaki et al. [5]. Interestingly in four patients 


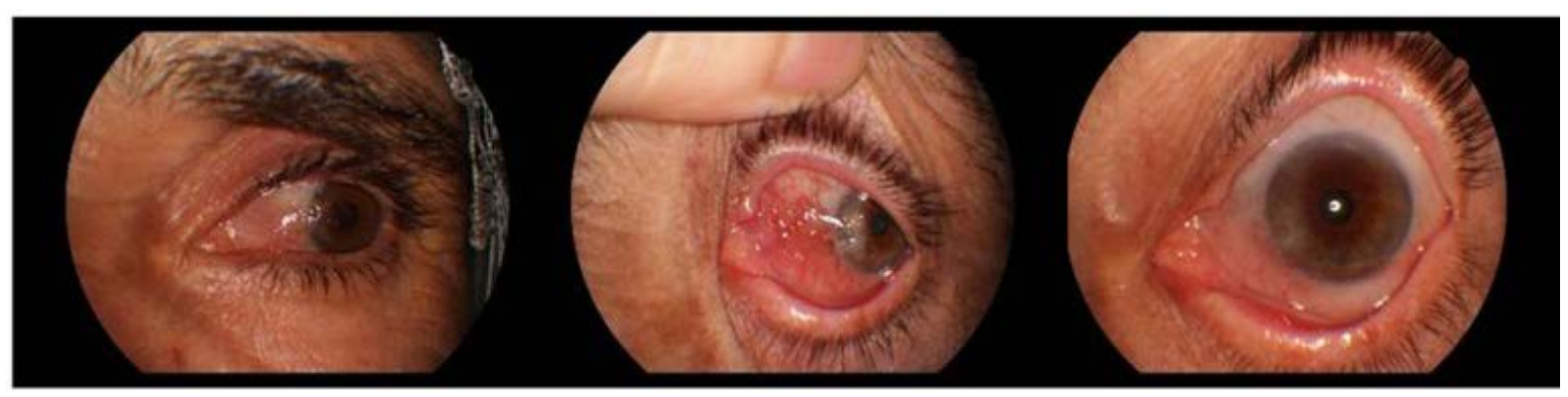

Figure 2. The clinical photograph of the patient are shown in Table 1 as fourth patient. Right, preoperative figure shows recurrent pterygium; Middle, early postoperative figure; Left, the eighth month postoperative figure.

with recurrences, schirmer test showed decreased tear secretion on the side of recurrence. All of four pterygiums were primary. However, tear secretion of the other side remained normal. Recurrence and asymmetric dry eyes disclosed an interesting association and raised some questions such as prevalence of asymmetric dry eye in pterygium so it needs of further investigation. Also atypia in one of the patient that resulted in very early recurrence is an interesting confirmation of Weinstein's report [18]. Although this study was conducted on limited number of participants, atypia was reported in one of the patients in the presented study that raises interesting questions such as the impact of atypia in recurrence of pterygium. It appears that ocular surface instability and atypia affected the outcome of this study unfavorably. However, no possible reason could be attributed to the recurrence observed in one patient (Table 1, third patient), who was young, aged twenty-eight years. An in-depth study of the VEGF isoforms may shed light onto the possible roles they play in recurrence. But conclusive evidence is still at large for this patient.

In analysing possible causes of high recurrence rate in this study, some questions can ensue such as inadequate post-operative medication, poor handling of limbal graft and in-appropriate size of the limbal graft. Yaisawang and Piyapattanakorn emphasized the importance of corticosteroids and encourage surgeons to use topical corticosteroids for enough time. Otherwise inadequate post-operative medication can cause high recurrence rate [19]. Clearly, we administered topical dexamethasone solutions for at least 45 days on a tapered regimen until conjunctival injection resolved and this regimen is definitely adequate. However Ti et al. emphasized that recurrence rates are inversely related to previous experience and simply poor handling of surgery can cause high recurrence rate [12], whereas it does not seems a causative factor of failing in hand of an experienced surgeon. We limited length of limbal graft according to the excised pterygium in head portion. Although the length between 4 or $6 \mathrm{~mm}$ and a width of $1.5 \mathrm{~mm}$ is a mini graft, it is an appropriate size for this technique because this size is enough large when compared with the component used in the limbal-conjunctival autograft so it can mimic the favorable effect of limbal component easily. Also we want to provide the effect of limbal stem cells in only the excised head portion. Besides to exclude the favorable impact of healthy conjunctiva on pterygium recurrence such as in conjunctival autograft that emphasized in some article, we did not excised tenon's layer from adjacent conjunctiva that dislocated to the midline [6] [11] [12]. Although limbal otograft, the adjacent tenon's layer may be the possible cause of high recurrence rate. This clue also mentioned in reports by Hirst [20]. He mentioned in his studies that transplantation of limbal stem cells was studiously avoided, seems to refute the need to deplete the superior limbus of their stem cells to achieve a low recurrence rate. The presented study confirms this suggestion from another way and in fact complements each other as such: he achieved low recurrence rate with extensive tenon's layer removal without transplantation of limbal stem cells whereas we failed in our technique by transplantation of limbal stem cells without tenon's layer removal [20].

As Hirst's mentioned the importance of extensive tenon's layer removal, it is well-known that the recurrence of pterygium is high when the underneath tenon's layer is not excised but this high recurrence rate was always shown in series without effect of limbal stem cells. The characteristic that distinguishes this work is that limbal stem cells on one side of pendulum whereas tenon's effect is on another side. So, the presented study proposes based on findings that tenon's layer is more determinative in recurrence than limbal stem cells, also widespread acceptance and high success of conjunctival autografting [9] [10] and/or limbal-conjunctival autograft probably due to excision of tenon's layer [7] [21] [22]. Limbal-conjunctival autograft results have been reported as successful as mitomycin C group [13] and Pulte reported conjunctiva-limbus autografts in pterygia have excellent 
efficacy against recurrence [21]. Probably conjunctival autograft adheres to episclera tightly and prevents fibrovascular growth up to forward.

Although this study has limited number of participants and lack of control group, it proved several points and complimentary indications such as atypia, asymmetric dry eye, effects of limbus in recurrent and multiple pterygia and effect of tenon's layer comparable to limbal stem cells. These are vital clues for the causes of recurrence. In this respect this study is unique.

\section{Conclusion}

In conclusion, from the above observations, it appears that pterygium is a complex disease with several underlying causative factors. The benefits associated with limbal graft are limited when applied alone. Instead, this study proposes a more comprehensive therapy into consideration. Conjunctival-limbal autograft, combining with extensive tenon's layer removal and if needed treatment for ocular surface instabilities, seems logical.

\section{Funding/Support}

None.

\section{Financial Disclosures}

None.

\section{The Contributions of the Authors}

The contributions of the authors are as follows: conception and design (SC, ED, LC, and OD), analysis and interpretation (SC, ED, LC, and OD), writing the article (SC), critical revision of the manuscript (SC, ED, LC, and OD), final approval of the manuscript (SC, ED, LC, and OD), data collection (SC), provision of the materials and patients (SC), literature search (SC), and administrative, technical, and logistical support (SC, ED, LC, and OD).

\section{Acknowledgements}

Suleyman Ciftci (SC): I had full access to all the data in the study, and take responsibility for the integrity of the data and the accuracy of the data analysis. Each of the coauthors (ED, LC, and OD) has seen and agrees with each of the changes made to this manuscript.

\section{References}

[1] Hirst, L.W. (2003) The Treatment of Pterygium. Survey of Ophthalmology, 48, 145-180. http://dx.doi.org/10.1016/S0039-6257(02)00463-0

[2] Reid, T.W. and Dushku, N. (2010) What a Study of Pterygia Teaches Us about the Cornea? Molecular Mechanisms of Formation. Eye Contact Lens, 36, 290-295. http://dx.doi.org/10.1097/ICL.0b013e3181eea8fe

[3] Kwok, L.S. and Coroneo, M.T. (1994) A Model for Pterygium Formation. Cornea, 13, 219-224. http://dx.doi.org/10.1097/00003226-199405000-00005

[4] Dushku, N. and Reid, T.W. (1994) Immunohistochemical Evidence That Human Pterygia Originate from an Invasion of Vimentin-Expressing Altered Limbal Epithelial Basal Cells. Current Eye Research, 13, 473-481. http://dx.doi.org/10.3109/02713689408999878

[5] Shimazaki, J., Yang, H.Y. and Tsubota, K. (1996) Limbal Autograft Transplantation for Recurrent and Advanced Pterygia. Ophthalmic Surgery Lasers \& Imaging, 27, 917-923.

[6] Al Fayez, M.F. (2013) Limbal-Conjunctival vs Conjunctival Autograft Transplant for Recurrent Pterygia: A Prospective Randomized Controlled Trial. JAMA Ophthalmology, 131, 11-16. http://dx.doi.org/10.1001/archophthalmol.2012.2599

[7] Gris, O., Güell, J.L. and del Campo, Z. (2000) Limbal-Conjunctival Autograft Transplantation for the Treatment of Recurrent Pterygium. Ophthalmology, 107, 270-273. http://dx.doi.org/10.1016/S0161-6420(99)00041-X

[8] Jaworski, C.J., Aryankalayil-John, M., Campos, M.M., et al. (2009) Expression Analysis of Human Pterygium Shows a Predominance of Conjunctival and Limbal Markers and Genes Associated with Cell Migration. Molecular Vision, 20, 2421-2434. 
[9] Vastine, D.W., Stewart, W.B. and Schwab, I.R. (1982) Reconstruction of the Periocular Mucous Membrane by Autologous Conjunctival Transplantation. Ophthalmology, 89, 1072-1081. http://dx.doi.org/10.1016/S0161-6420(82)34681-3

[10] Kenyon, K.R., Wagoner, M.D. and Hettinger, M.E. (1985) Conjunctival Autograft Transplantation for Advanced and Recurrent Pterygium. Ophthalmology, 92, 1461-1470. http://dx.doi.org/10.1016/S0161-6420(85)33831-9

[11] Riordan-Eva, P., Kielhorn, I., Ficker, L.A., McG Steele, A.D. and Kirkness, C.M. (1993) Conjunctival Autografting in the Surgical Management of Pterygium. Eye, 7, 634-638. http://dx.doi.org/10.1038/eye.1993.146

[12] Ti, S.E., Chee, S.P., Dear, K.B. and Tan, D.T. (2000) Analysis of Variation in Success Rates in Conjunctival Autografting for Primary and Recurrent Pterygium. British Journal of Ophthalmology, 84, 385-389. http://dx.doi.org/10.1136/bjo.84.4.385

[13] Güler, M., Sobaci, G., Ilker, S., Oztürk, F., Mutlu, F.M. and Yildirim, E. (1994) Limbal-Conjunctival Autograft Transplantation in Cases with Recurrent Pterygium. Acta Ophthalmologica, 72, 721-726. http://dx.doi.org/10.1111/j.1755-3768.1994.tb04688.x

[14] Zheng, K., Cai, J., Jhanji, V. and Chen, H. (2012) Comparison of Pterygium Recurrence Rates after Limbal Conjunctival Autograft Transplantation and Other Techniques: Meta-Analysis. Cornea, 31, 1422-1427. http://dx.doi.org/10.1097/ICO.0b013e31823cbecb

[15] Mutlu, F.M., Sobaci, G., Tatar, T. and Yildirim, E. (1999) A Comparative Study of Recurrent Pterygium Surgery: Limbal Conjunctival Autograft Transplantation versus Mitomycin C with Conjunctival Flap. Ophthalmology, 106, 817-821. http://dx.doi.org/10.1016/S0161-6420(99)90172-0

[16] Singh, G., Wilson, M.R. and Foster, C.S. (1990) Long Term Follow up Study of Mitomycin Eye Drops as Adjunctive Treatment for Pterygia and Its Comparison with Conjunctival Autograft Transplantation. Cornea, 9, 331-334. http://dx.doi.org/10.1097/00003226-199010000-00011

[17] Hirst, L.W., Sebban, A. and Chant, D. (1994) Pterygium Recurrence Time. Ophthalmology, 101, 755-758. http://dx.doi.org/10.1016/S0161-6420(94)31270-X

[18] Weinstein, O., Rosenthal, G., Zirkin, H., Monos, T., Lifshitz, T. and Argov, S. (2002) Overexpression of p53 Tumor Suppressor Gene in Pterygia. Eye, 16, 619-621. http://dx.doi.org/10.1038/sj.eye.6700150

[19] Yaisawang, S. and Piyapattanakorn, P. (2003) Role of Post-Operative Topical Corticosteroids in Recurrence Rate after Pterygium Excision with Conjunctival Autograft. Journal of the Medical Association of Thailand, 86, 215-223.

[20] Hirst, L.W. (2008) Prospective Study of Primary Pterygium Surgery Using Pterygium Extended Removal Followed by Extended Conjunctival Transplantation. Ophthalmology, 115, 1663-1672. http://dx.doi.org/10.1016/j.ophtha.2008.03.012

[21] Pulte, P., Heiligenhaus, A., Koch, J., Steuhl, K.P. and Waubke, T. (1998) Long-Term Results of Conjunctiva-Limbus Autografts in Patients with Pterygia. Klinische Monatsblätter für Augenheilkunde, 213, 9-14. http://dx.doi.org/10.1055/s-2008-1034937

[22] Koch, J.M., Mellin, K.B. and Waubke, T.N. (1990) Initial Experience with Autologous Conjunctiva/Limbus Transplantation in Pterygium. Klinische Monatsblätter für Augenheilkunde, 197, 106-109. http://dx.doi.org/10.1055/s-2008-1046250 